\title{
El atraso escolar en las escuelas multigrado de las comunidades del municipio de Molango
}

de Escamilla, Hidalgo

\author{
Ángeles Olvera Gabriela Anahí ${ }^{1}$ \\ García Razo Liliana Lizbeth ${ }^{2}$ \\ Lara Ramírez Aurelia Eugenia ${ }^{3}$ \\ Machorro Valdez César Rubén ${ }^{4}$ \\ Asesora: Dra. Esther Fragoso Fernandez
}

\section{Introducción}

El artículo 3o de la Constitución Política de los Estados Unidos Mexicanos de 1917, indica que la educación es un derecho de toda persona nacida en territorio mexicano. Por tal motivo es importante la preocupación por factores que obstaculizan el desarrollo de la educación básica. Gimeno Sacristán (1988) plantea como principales preocupaciones psicopedagógicas y sociales, dentro del curriculum al fracaso escolar, la desmotivación de los alumnos, el tipo de relaciones entre éstos y los profesores, la indisciplina en clase, entre otros.

Tal como lo plantea Josefina Zoraida Vázquez Los pocos que se atreven a cultivarla [la historia de la educación] tienen que desafiar el legado que han dejado otras obras tradicionales que hacían una crónica de ideas pedagógicas, fundación de instituciones y ennumeración de leyes educativas, a las que se solía agregar números de alfabetizados, alumnos y maestros que no tenían otros lectores que los pobre estudiantes que los tenía que utilizar como libros de texto. Los mejores por lo menos relacionaban los acontecimientos educativos con los cambios filosóficos que los habían influenciado (Vázquez, 1992 p. IX).

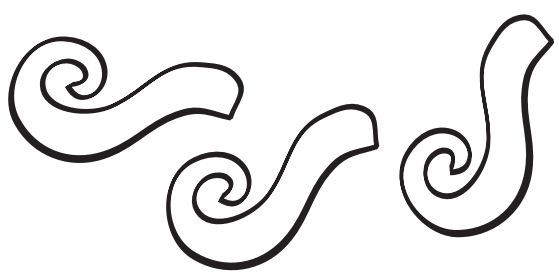

\section{Planteamiento del problema}

Las principales causas educativas del atraso escolar en los estudiantes del cuarto y quinto grado de primaria en las comunidades con escuelas multigrado del municipio de Molango, Hidalgo

\section{Objetivo general}

Conocer las causas principales por la que los estudiantes de cuarto grado y quinto grado de primaria presentan atraso escolar, tomando en cuenta la educación que reciben dentro de la escuela como en la sociedad.

\section{Objetivos específicos}

- Conocer las principales características del alumno que presenta atraso escolar.

- Conocer las estrategias que utilizan en la Institución para trabajar con los alumnos que manifiestan dicho problema.

\section{Preguntas de investigación}

- ¿Cuáles son las principales características de un alumno que presenta atraso escolar?

1 Octavo semestre de la Licenciatura en Ciencias de la Educación. (ga-ang@hotmail.com) 2 Octavo semestre de la Licenciatura en Ciencias de la Educación. (deelishis_liliz@hotmail.com)

3 Octavo semestre de la Licenciatura en Ciencias de la Educación. (iamsepa@ $@$ hotmail.com)

4 Octavo semestre de la Licenciatura en Ciencias de la Educación. (cesmava88_20@live.com.mx) 
- ¿Cuáles son las estrategias que se utilizan para trabajar con los alumnos que presentan dicho problema?

- ¿Qué sugerencias conviene proponer a los docentes para ayudar a sus alumnos a prevenir y abatir el atraso escolar?

\section{Justificación}

La calidad de la educación es una urgencia propuesta en el Proyecto Educativo Regional Lasallista Latinoamericano (PERLA).

El Proyecto PERLA declara cinco urgencias en materia educativa, que deben ser atendidas a la luz de los valores y el carisma lasallistas.

Para fines de esta investigación cualitativa, se dará énfasis en la urgencia tres, la cual trata sobre la Educación de Calidad. Propone que la pedagogía lasallista debe adaptarse a la época en que los jóvenes vivan, para prepararlos para ocupar un puesto en la sociedad, mediante una formación humana de calidad.

Tomando en cuenta la urgencia mencionada, se considera importante la necesidad de investigarlas causas del atraso escolar en los alumnos de cuarto grado de primaria, residentes en las comunidades del municipio de Molango de Escamilla, Hgo. Se trabajará con alumnos de este nivel de primaria porque es conveniente para saber cómo ha sido su historial en los años que lleva estudiando la primaria y qué planes tiene para futuro en cuanto a la culminación de sus estudios en este nivel básico. Se pretende el involucramiento en el contexto, para conocer la forma de educación que se lleva a cabo, tanto en la escuela primaria en cuarto y quinto, así como en la sociedad, en dicho escenario. En medida de lo posible, es factible aportar sugerencias de mejora para prevenir y corregir el atraso escolar.

Los mayores beneficios con este estudio cuali- tativo son una mejor calidad educativa para los alumnos de cuarto grado de primaria en el municipio de Molango y en las comunidades de Pemuxtitla, Ixmolintla, Santiago, Chalma y Tenango, así como la continuidad para la formación en investigación de los alumnos de Ciencias de la Educación, quienes fungirán como investigadores de campo.

Se tiene conciencia de la situación que viven las comunidades rurales en Hidalgo, y de la deficiencias educativas que existen, es por ello que se muestra interés en indagar de manera natural y vivencial, el contexto en el que se desarrollan los estudiantes objeto de estudio, y así conocer las expectativas que los estudiantes de este nivel tienen para el futuro y la influencia que tienen sus familias sobre ellos en su desempeño escolar y en sus decisiones de vida.

\section{Marco histórico}

A inicios del siglo $X X$, se propuso la definición de atraso o retraso escolar. Según Granja, "retrasados escolares fue una referencia de clasificación en la que se incluían por igual a niños 'desaplicados', 'inatentos', 'tontos' y 'mal intencionados' que tenían en común no avanzar en sus estudios y quedar constantemente rezagados" (Granja, 2009, p. 2). Se subestimaba las capacidades diferentes que podían presentar ciertos alumnos y los déficits o carencias de otros. A todos estos niños que presentaban una situación parecida, se les englobaba dentro del término de retraso escolar.

La definición del médico francés René Crochet, tuvo gran impacto en nuestro país a inicios de siglo, sobre todo, en los tratados de higiene y salud y en los cursos para maestros. La definición es la siguiente: "retardado o retrasado escolar es todo niño que desde el punto de vista escolar está retrasado 2 o 4 años en relación a la media escolar de los niños de su edad" (Granja, 2009, p. 2). 


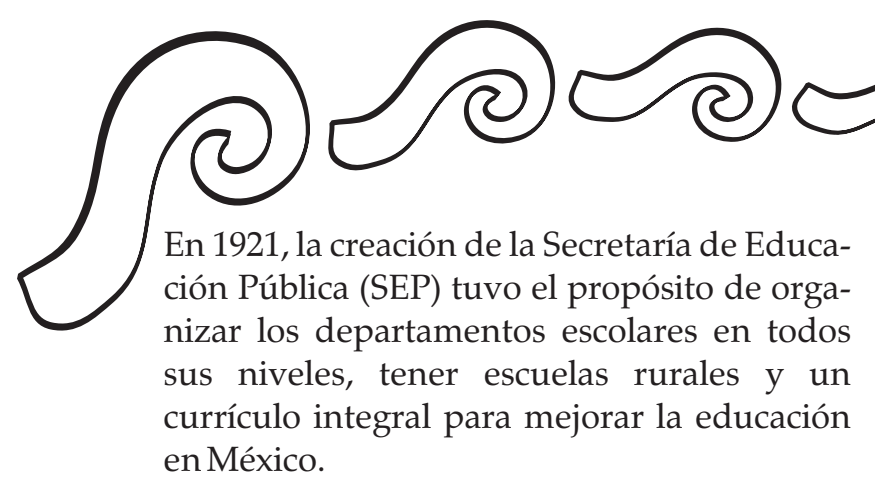

Al fungir el cargo de Secretario de Educación Pública, José Vasconcelos asumió tareas educativas con la idea de vincular la escuela con la realidad social. Él afirmó:

$\mathrm{Al}$ decir educación me refiero a una enseñanza directa de parte de los que saben algo, en favor de los que nada saben; me refiero a una enseñanza que sirva para aumentar la capacidad productiva de cada mano que trabaja, de cada cerebro que piensa [...] Trabajo útil, trabajo productivo, acción noble y pensamiento alto, he allí nuestro propósito [...] Tomemos al campesino bajo nuestra guarda y enseñémosle a centuplicar el monto de su producción mediante el empleo de mejores útiles y de mejores métodos. Esto es más importante que distraer los en la conjugación de los verbos, pues la cultura es fruto natural del desarrollo económico [...] (Secretaría de Educación Pública, 2011).

A partir de este proyecto comienza un cambio y un avance dentro de la educación, el cual hoy en día se encuentra estancado, el problema del atraso escolar y la educación tradicional son vigentes, las escuelas tienen herramientas insuficientes para una educación de calidad; el presupuesto económico que designa gobierno es escaso y peor aún, en las escuelas no hay maestros suficientes para la cantidad de estudiantes que existen.

Hoy en día, según la Organización para la Cooperación y Desarrollo Económicos (OCDE), México pierde 25 mil millones de dólares al tener a 50 por ciento de sus estudiantes de 15 años en los últimos niveles de desempeño escolar. Según Silvia Schmelkes, citada por Karina Avilés (2011), "la relación perversa entre la Secretaría de Educación Pública y el Sindicato Nacional de Trabajadores de la Educación se encuentra en la base de cualquier reforma educativa, por lo que se necesita definir nuevas reglas para el funcionamiento del sistema" (Avilés, 2011).

\section{Marco conceptual}

Atraso: Falta de desarrollo, o desarrollo inferior al normal (Farlex, s.f.)

* Propia definición de atraso: Desarrollo inferior al estándar o promedio.

Escolar: Relativo al estudiante o a la escuela (Farlex, s.f.)

* Propia definición de escolar: Propio de la escuela o a la institución escolar.

Enseñanza: Comunicación de conocimientos, habilidades, ideas o experiencias a una persona que no las tiene con la intención de que las comprenda y haga uso de ellas. (Farlex, s.f.)

* Propia definición de enseñanza: Proceso de dar a conocer ciertos contenidos con el fin de la construcción del conocimiento, mediante la mediación del docente, de los materiales y de los instrumentos.

Escuela: Establecimiento donde se da cualquier género de instrucción. (Definicion.de diccionario, s.f.)

* Propia definición de escuela: Todo recinto donde se lleva a cabo el acto educativo formal y no formal.

Educación: Proceso de socialización de los individuos. Al educarse, una persona asimila y aprende conocimientos. La educación también implica una concienciación cultural y 


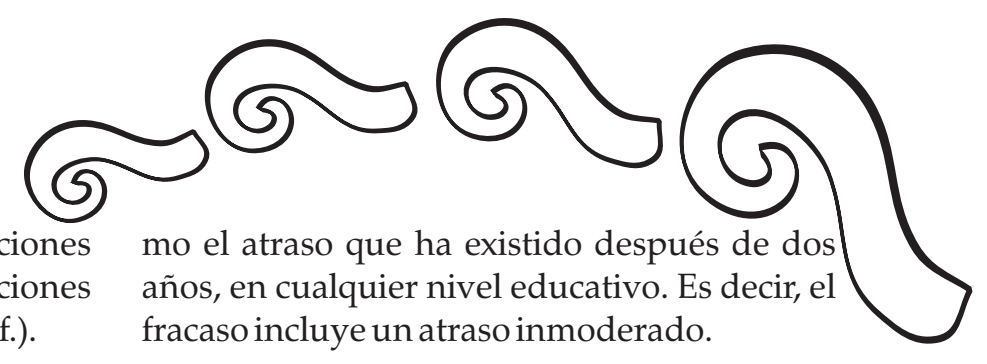

* Propia definición de educación: Es un proceso permanente en el que se da la construcción intencional de conocimientos por medio del descubrimiento, la exploración, la comparación, el análisis, la síntesis, entre otras funciones mentales.

Educación rural: Se refiere a la educación que hace referencia a lo perteneciente o relativo a la vida en el campo(Definicion de diccionario, s.f.).

*Propia definición de rural: Referente a la vida en los lugares no urbanizados.

Rezago Educativo: El rezago educativo es la condición en la que se encuentra una persona mayor de 15 años cuando no ha concluido su enseñanza básica. (Núñez, 2005,)

* Propia definición de rezago educativo: Relacionado con las personas que no concluyeron su educación básica y que son mayores a quince años.

Para fines de esta investigación, entenderemos como atraso escolar al estancamiento de máximo dos años del estudiante escolarizado formalmente, con respecto a la media de los alumnos de su edad y desarrollo correspondiente. Si sobrepasa los dos años el estancamiento, se le puede llamar fracaso escolar.

\section{Marco teórico}

El atraso o retraso escolar se ha dado a través del tiempo como un fenómeno desarrollado en el ambiente escolarizado formal, siendo éste uno de los principales problemas en la educación formal contemporánea.

Según el portal electrónico de psicopedagogía.com, frecuentemente se confunde el término "atraso" con "fracaso", siendo este últi-

Los autores del portal previamente mencionado consideran que el atraso escolar ha pasado de ser considerado como resultado de la pereza del alumno a ser una deficiente formación neurofisiológica para llegar a un concepto sociológico, referente a la inadaptación dentro de la estructura escolar. Dentro de esta inadaptación influyen tres factores determinantes: el alumno, la escuela y la familia. Según dichos autores descarta, entonces, la responsabilidad única del alumno, ya que el contexto tiene vital importancia pues son las dos organizaciones en las que está inmerso la mayor parte del tiempo. Se proponen los siguientes factores:

Factores familiares: La dinámica familiar y el nivel socioeconómico influyen en el desempeño escolar del educando, siendo ésta, parte importante para la estabilidad emocional del alumno.

Factores escolares: Influyen tanto los factores formales como horarios, estructura escolar, ideología, así como los contenidos que se abordan en cada materia con un determinado método pedagógico.

Factores del propio niño: Tal como su capacidad y estilo de aprendizaje, desarrollo neurofisiológico y motivación intrínseca y extrínseca. Se puede analizar detenidamente, en este aspecto, los siguientes elementos:

- Debilidad mental: Aunque este elemento obstaculiza el progreso del alumno, "la debilidad ligera o límite no suele ser la causa principal del fracaso escolar" (Portal de psicopedagogía, S/A, (s.f.).

- Rechazo escolar: Puede ser que el alumno se oponga a toda adquisición de conocimiento por parte de la escuela, como resultado de una inhibición de una problemática. 


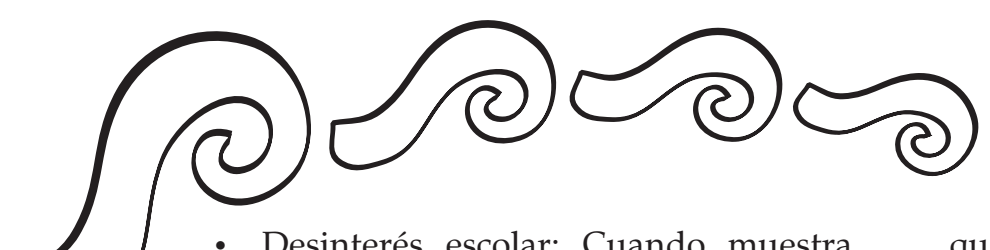

- Desinterés escolar: Cuando muestra aversión por todo lo que esté relacionado con la escuela, así como la manifestación de aburrimiento.

- Inhibición escolar: Cuando el alumno no muestra la capacidad de concentrarse en una tarea determinada, así como cuando se le diagnostica con déficit de atención.

Puede deberse también a cambios hormonales, característicos de la superación de una etapa biológica y la entrada a una nueva, así como la influencia social que conllevan las etapas de madurez en los niños y jóvenes, estudiantes de la educación básica.

Es posible realizar un diagnóstico basándose en resultados psicométricos y entrevistas directas con el niño a estudiar, pues la buena interpretación de sus actitudes y comportamientos pueden ser indicadores de lo que puede causar un atraso escolar.

\section{Marco referencial}

El tema del rezago escolar es multifactorial cuya importancia a dado como resultado diversas investigaciones, entre la que se encuentra aquella que fue realizada en Chihuahua y la zona metropolitana de la ciudad de México.

Los resultados de dicha investigación fueron publicados en el artículo titulado Centro de investigación básica intensiva: una alternativa al rezago escolar se comenta lo siguiente:

A partir de los estudios realizados por la SEP para sustentar el plan nacional de educación del sexenio 1976-1982, se decidió establecer tres programas prioritarios para el sector educativo en su conjunto: educación primaria, de adultos e indígena. Para llevar a cabo el objetivo central de la primera prioridad señalada, se creó el programa " primaria para todos los niños", que pretendía lograr que para septiembre de 1980 todos los niños entre 6 y 14 años de edad tuvieran la posibilidad de cursar la educación primaria y permanecer en ella hasta terminarla (Lavín, 2005, p.36).

En este contexto, el equipo conformado para llevar a cabo este programa se dio a la tarea de plantear distintas alternativas diversificadas de atención a la población rezagada.

El análisis de las estadísticas de la población inscrita por edad y por grado en las escuelas primarias del país denotaba la presencia de un fenómeno constante a lo largo de las distintas cohorte generacionales: aparentemente la población inscrita de 8 y 9 años abarcaba a la casi totalidad de la población existente de esas edades, pero el nivel de atención bajaba considerablemente a partir de los 10 años de edad, dejando un importante grupo de niños sin atender entre los 10 y 14 años. Este fenómeno evidentemente representa la presencia de un agudo proceso de rezago escolar y de deserción que era necesario abordar en su génesis, pero a la vez abría la tarea de establecer una modalidad de atención específica para estos niños, que tuviera la capacidad de adecuarse a su momento y a sus circunstancias.

Fue entonces cuando se gestó el modelo de educación básica intensiva para niños desertores de 11 a 14 años de zonas urbanas marginadas, cuyo diseño, bajo ciertos lineamientos generales, fue encomendado al Departamento de Investigaciones Educativas del IPN(DIEIPN), bajo la dirección de Gabriel Cámara, quien imprimió al modelo su carácter participativo, flexible intensivo y descentralizado. Paralelamente, se inició su implementación en la ciudad de Chihuahua, a cargo del Centro de Estudios Generales, A.C., con la asesoría del DIE."

De marzo del año 1979 a diciembre de 1980, se realizó el diseño de la experiencia bajo la modalidad de investigación-acción, en ella participaron maestros y promotores ubicados en las 


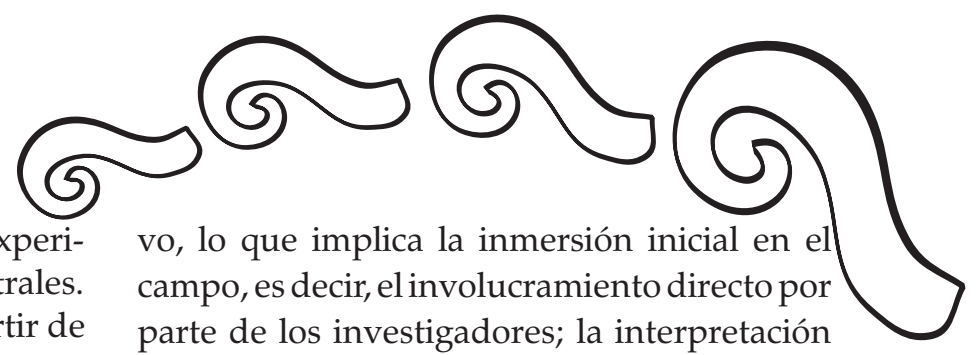

colonias seleccionadas para iniciar la experimentación junto con los equipos centrales. Los problemas que se presentaron a partir de la modalidad de contratación de los instructores llevaron a la SEP a cerrar los denominados centros del área metropolitana de la ciudad de México, Por otra parte, Chihuahua continúo los trabajos y logró certificar a fines del año 1981sus primeros egresados; a partir de este mismo año, la SEP encomendó a la CONAFE proseguir con esta experiencia, en la que se incluyeron grupos de niños de 9 a 14 años y niños del medio rural.

Y es a través del proceso de evaluación que se verificó que el programa respondiera a las necesidades de la población a atender, observándose altos niveles de asistencia y un rendimiento escolar similar o superior al de los alumnos de escuelas federales de un nivel socioeconómico semejante. Trabajándose con los denominados grupos locales.

Es en 1984 que se cambió a los Grupos Locales por las instancias administrativas estatales. Debido a la insuficiente infraestructura de apoyo pedagógico y de promoción, así como al cambio del grupo de instructores es que finalmente en 1985 se cancela el programa.

\section{Enfoque}

El enfoque de la presente investigación es de corte cualitativo, por lo que se estudiará el contexto desde una perspectiva interna, comprendiendo los diversos factores que estructuran a la población objeto de estudio, así como el análisis de las condiciones de vida del municipio de Molango y de las comunidades de Pemuxtitla, Ixmolintla, Chalma y Tenango. Lo anterior con el fin de indagar acerca del atraso escolar en los alumnos de cuarto grado de primaria.

Por su carácter de cualitativa, la investigación será realizada con base en un proceso inducticaracterísticas concretas del entorno; la flexibilidad, puesto que la investigación cualitativa debe ser tolerante a los cambios e imprevistos; se da cabida a preguntas como fuente esencial de la investigación y la recolección de datos, mediante los instrumentos diseñados fortuitamente, según los recursos disponibles, para poder conocer la situación de atraso escolar en las comunidades aledañas a Molango.

\section{Alcance}

El alcance más apropiado para realizar esta investigación es de carácter descriptivo, pues se trata de dar una mayor estructuración a la investigación cualitativa, describiendo los factores y características por los que se produce el fenómeno del atraso escolar, así como la proposición de medios de mejora en la comunidad. Se pretende entender el fenómeno a estudiar, en este caso, en los alumnos de cuarto grado de primaria.

\section{Diseño}

El diseño de la investigación es no experimental, implica buscar e indagar en el contexto tal y como se dan los sucesos naturalmente. No se pretende alterar, el ambiente, sólo estudiar qué y cómo pasa, por medio de la observación y la interacción directa con los miembros de las comunidades objeto de estudio.

Es una investigación con diseño transversal, es decir, que utilizará la descripción, la explicación y la correlación de las variables de los fenómenos que se estudiarán. Todo será dentro de una misma época y un mismo espacio, por lo que se tratará de ahondar en las situaciones que sucedan en el ambiente, para su análisis. 


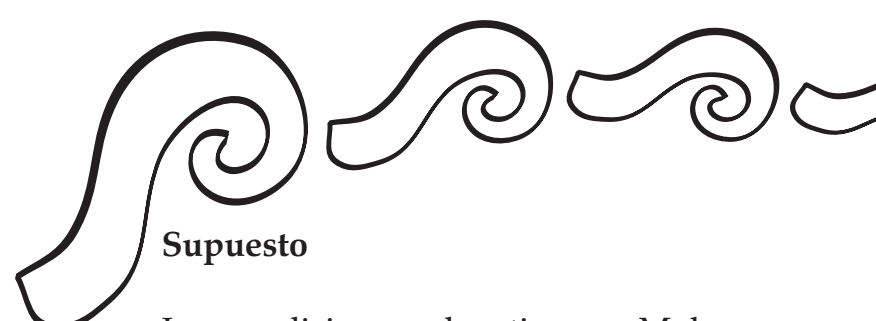

Las condiciones educativas en Molango presentan carencias para desarrollar óptimamente el proceso de Enseñanza - Aprendizaje. Esto con base en el conocimiento de las condiciones educativas de nuestro Estado y, en particular, de las comunidades de la sierra, inferencialmente. El atraso escolar es una limitante para llevar a cabo dicho proceso, pues las condiciones dentro de la escuela como la relación con sus compañeros y maestro- y en su familia -como el tipo de familia (monoparental, disfuncional) y el apoyo que reciben para continuar estudiando- orillan a desertar por algún tiempo o tener un bajo nivel de rendimiento académico, por lo que se da dicho fenómeno con mucha frecuencia entre los alumnos de primaria en nuestro país.

\section{Población}

La población a estudiar, serán los niños que estén estudiando el cuarto grado de primaria en las escuelas de las comunidades de Tenango, Chalma, Ixmomiltla, y Pemuxtitla del municipio de Molango, Hidalgo.

Comunidad de Tenango: está situada en el Municipio de Molango de Escamilla (en el Estado de Hidalgo). Tiene 378 habitantes. Tenango está a 1540 metros de altitud. (Pueblos américa, s.f.).Comunidad de Chalma: No se encontraron enlaces en internet para obtener información de esta comunidad.Comunidad de Ixmolintla: está situado en el Municipio de Molango de Escamilla (en el Estado de Hidalgo). Tiene 253 habitantes. Ixmolintla está a 1720 metros de altitud. (Pueblos américa, s.f.).Comunidad de Pemuxtitlase localiza en el Municipio Molango de Escamilladel Estado de HidalgoMéxico y se encuentra en las coordenadas GPS:Longitud (dec): -98.763056, Latitud (dec): 20.831667. La localidad se encuentra a una mediana altura de 1300 metros sobre el nivel del mar.
La Población total de Pemuxtitla es de 240 personas, de cuales 124 son masculinos y 116 femeninas. Los ciudadanos se dividen en 97 menores de edad y 143 adultos, de cuales 47 tienen más de 60 años. Estructura social: Derecho a atención médica por el seguro social, tienen 6 habitantes de Pemuxtitla. Estructura económica: En Pemuxtitla hay un total de 59 hogares.De estas 59 viviendas, 9 tienen piso de tierra y unos 5 consisten de una sola habitación. 52 de todas las viviendas tienen instalaciones sanitarias, 17 son conectadas al servicio público, 44 tienen acceso a la luz eléctrica. La estructura económica permite a 0 viviendas tener una computadora, a 11 tener una lavadora y 14 tienen una televisión. Educación escolar en Pemuxtitla: Aparte de que hay 22 analfabetos de 15 y más años, 0 de los jóvenes entre 6 y 14 años no asisten a la escuela. De la población a partir de los 15 años 21 no tienen ninguna escolaridad, 86 tienen una escolaridad incompleta. 30 tienen una escolaridad básica y 22 cuentan con una educación post-básica. Un total de 16 de la generación de jóvenes entre 15 y 24 años de edad han asistido a la escuela, la mediana escolaridad entre la población es de 5 años. (Nuestro México, s.f.,) Estos niños objeto de nuestro estudio, habitan en comunidades rurales. Dichas poblaciones tienen carencias tanto en infraestructura como en economía. Son hijos de familias con un nivel socioeconómico bajo, dedicadas, en su mayoría al campo $\mathrm{y}$ al comercio, lo cual influye fuertemente en el aspecto educativo. Los niños de cuarto grado de primaria tienen entre 8 a 10 años de edad, con un desarrollo cognitivo en la etapa de operaciones concretas, según Piaget. Tienen habilidad discursiva y también de clasificación, análisis y síntesis.

\section{Muestra}

Nuestra muestra serán niños de cuarto grado y quinto grado de primaria que sean asignados de acuerdo a la disponibilidad del grupo, en las comunidades de Pemuxtitla, Ixmolintla, 


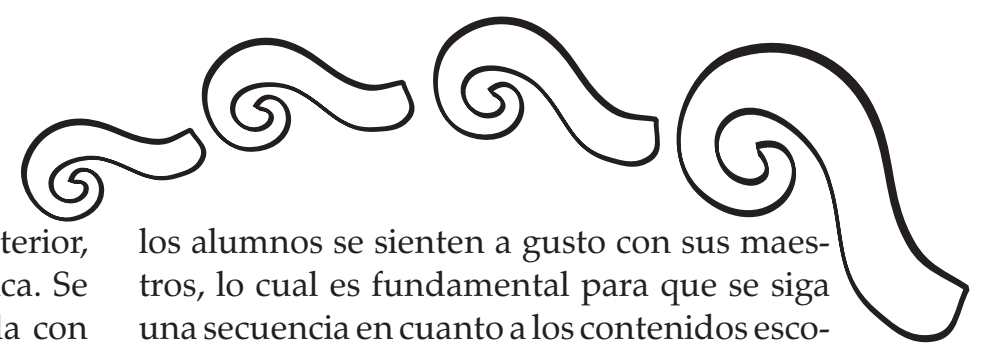

Chalma y Tenango. Atendiendo a lo anterior, se trata de una muestra no probabilística. Se busca trabajar de manera personalizada con ellos, al participar en la actividad los cuatro miembros del equipo de investigación. Así se podrán atender personalmente las necesidades que muestren, principalmente detectar a qué se debe su atraso escolar.

\section{Resultados}

En las comunidades pertenecientes a Molango, tales como Tenango, Chalma, Ixmolintla y Pemuxtitla se aplicaron dos instrumentos de medición, los cuales fueron entrevistas e historias de vida. Se recopiló información de 39 niños en las comunidades previamente mencionadas. Para fines de esta investigación, la cual tiene como tema principal al atraso escolar en comunidades del municipio de Molango, Hidalgo, tomaremos en cuenta la definición de "atraso escolar" ubicada en el marco conceptual.

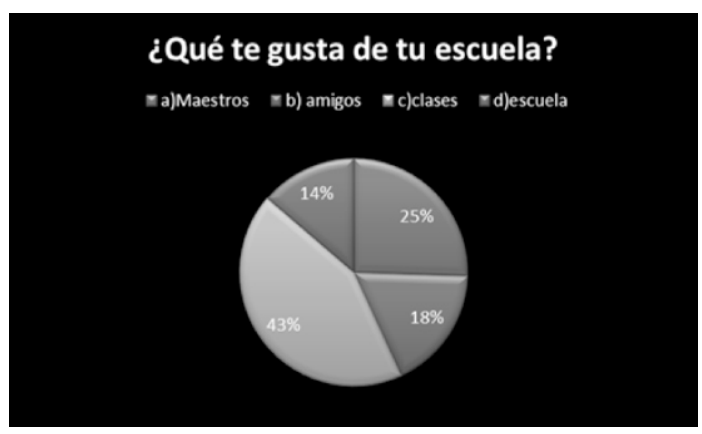

Gráfica 1

El $43 \%$ de los alumnos entrevistados dicen que les agradan sus clases. Uno de los factores que detonan el atraso escolar, es el desinterés por parte de los alumnos por tomar las clases. Esto es, entonces, un resultado favorable, pues con esto se demuestra que la mayoría de los alumnos no sufren de atraso escolar por esta causa.

$25 \%$ de los alumnos afirmaron que lo que más les gusta de su escuela son sus maestros. Lo anterior demuestra que una tercera parte de

lares. Se pudo observar que la relación que el maestro lleva con el grupo es de respeto y confianza, y en una de las escuelas existe un apoyo extra escolar para los alumnos con dificultades.14\% de los alumnos mencionan que les gusta su escuela, les agrada y se sienten cómodos y contentos dentro del contexto escolar en el que se encuentran. Los establecimientos asisten los alumnos a tomar clases, carecen de algunos materiales, sin embrago los alumnos aprovechan la oportunidad de ir a la escuela.18\% de los alumnos mencionan que lo que más les gusta de su escuela son sus amigos, se observó la existencia de buenas relaciones entre los alumnos. Son solidarios y saben trabajar en equipo, siempre con la ayuda de sus docentes. Se puede hacer la inferencia de que existe mínima exclusión entre los alumnos, lo cual se da en las escuelas dentro de un contexto urbanizado.

Es importante mencionar que el porcentaje de las respuestas brindadas puede variar a lo que se menciona con anterioridad, pues varios entrevistados en diferentes ocasiones respondieron con más de una opción.

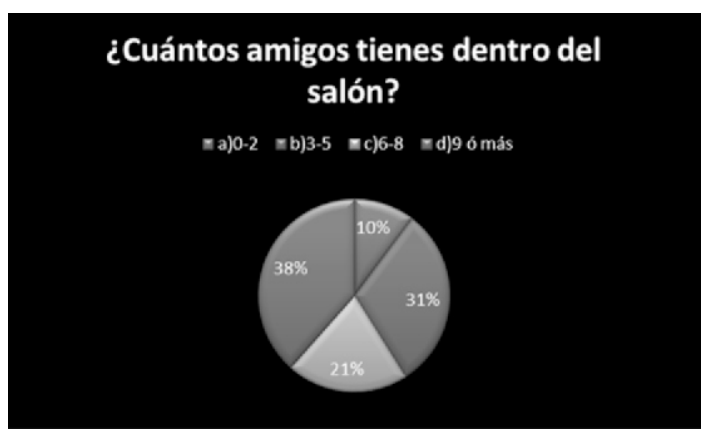

Gráfica 2

En la siguiente gráfica se muestran los porcentajes a la pregunta de cuantos amigos tiene el entrevistado dentro del salón, el 38\% de los entrevistados dice que tiene 9 amigos o más, lo cual es indicativo que hay una buena relación en todo el grupo ya que no hay grupos de más 

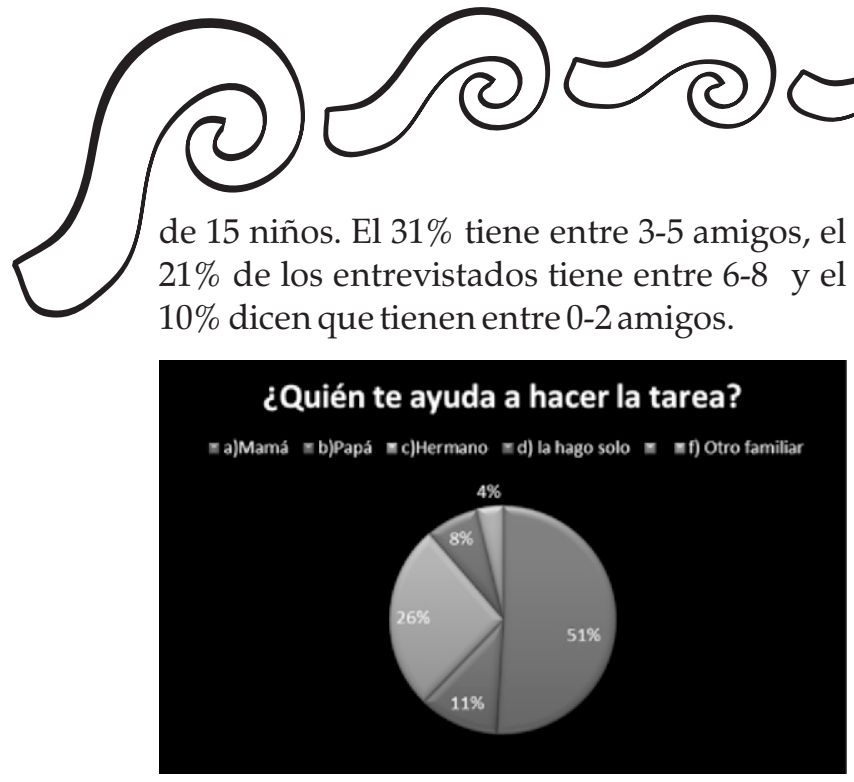

Gráfica 3

En la gráfica anterior se muestran los resultados a la tercera pregunta donde se les cuestionó a los entrevistados quienes le ayudaban a hacer la tarea, la mayoría con un $51 \%$ de las respuestas dicen que su mamá les ayuda seguido por el $26 \%$ que dicen que reciben ayuda de un hermano(a), el $11 \%$ recibe ayuda de su papá, el $8 \%$ hace solo su tarea y el $4 \%$ lo ayuda otro familiar. La figura materna parece que está más presente dentro de las familias de las comunidades de Molango.

La siguiente gráfica muestra quiénes dan dinero dentro de la casa. El 56\% de los entrevistados mencionan que su papá es el que trabaja y es el que lleva dinero a la casa le sigue con un $31 \%$ la mamá que en su mayoría son labores de limpieza, el $7 \%$ dicen que otro familiar es el que lleva dinero mientras que el $6 \%$ dice que su hermano lleva dinero a la casa. Cabe mencionar que ninguno de los entrevistados trabaja, (algunos eligieron más de una respuesta).

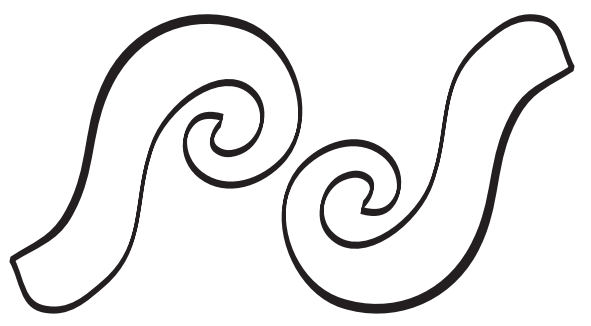

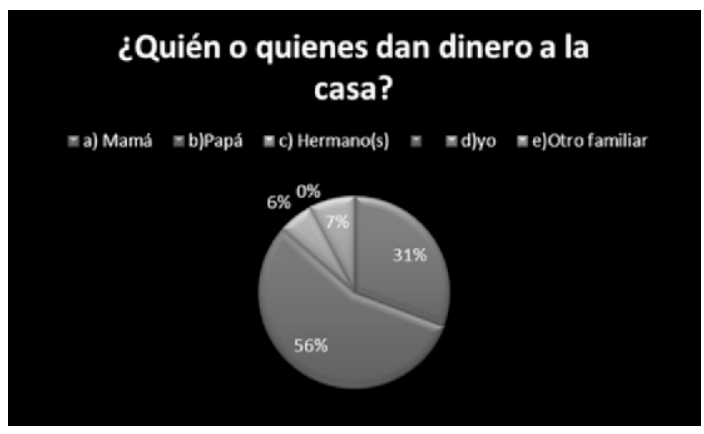

Gráfica 3

Con base en el análisis anterior, se encontraron porcentajes bajos en cuanto al índice de reprobación en los alumnos de cuarto y quinto grado de las comunidades visitadas en el municipio de Molango de Escamilla, Hidalgo. Se encuentra con un $10 \%$ de retraso escolar debido al contexto familiar en el que se encuentran o bien, estuvieron en los años escolares que recusaron, ejemplo de ellos son la ausencia de miembros de su familia a causa de la migración, falta de recursos económicos, enfermedades, y trabajos pesados en su mayoría el comercio y el campo.

Por otro lado, encontramos que el $90 \%$ restante son alumnos regulares los cuales no han tenido necesidad de recursar ciclos escolares pues afortunadamente cuentan con apoyos como el programa de oportunidades y gracias a que el gobierno federal ha puesto en marcha nuevas reformas educativas en donde se obligan a equipar íntegramente a las escuelas, esto hace que en los alumnos exista el interés de continuar estudiando.

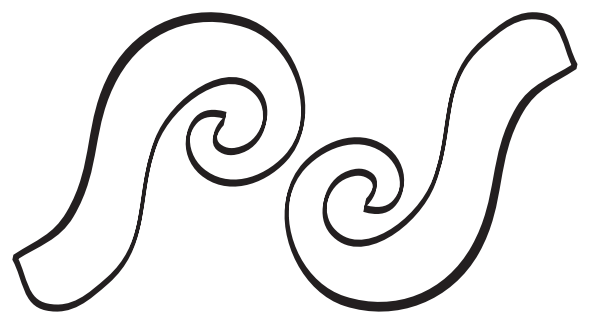




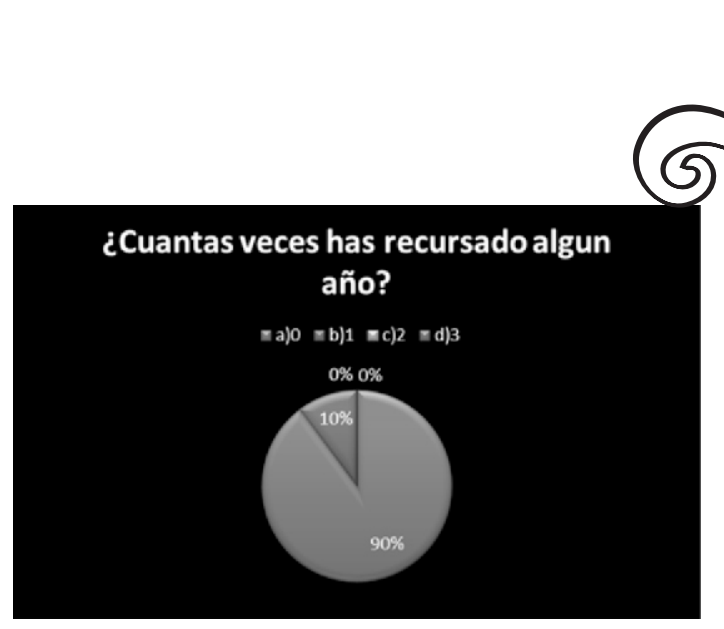

Gráfica 5

Relacionado con la pregunta anterior se le preguntó al alumno de quienes pudieron haber influido para que el perdiera el año, de los 4 que lo han hecho 2 consideran que fue su culpa, uno a causa de sus padres y otro por culpa de sus amigos.

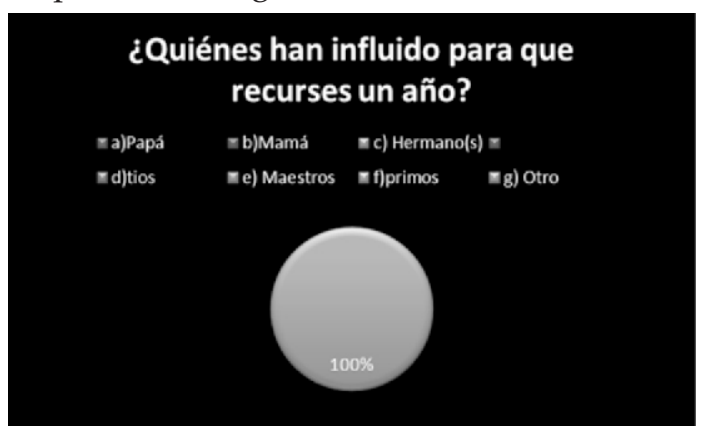

Gráfica 6

En la séptima gráfica se muestran los porcentajes de según los entrevistados, quien les ayuda cuando tienen una dificultad de carácter académico. El $62 \%$ recibe ayuda de sus maestros, el $17 \%$ de sus padres, el $15 \%$ recibe ayuda de sus compañeros, el $4 \%$ de sus hermanos y $2 \%$ recibe ayuda de otra persona.

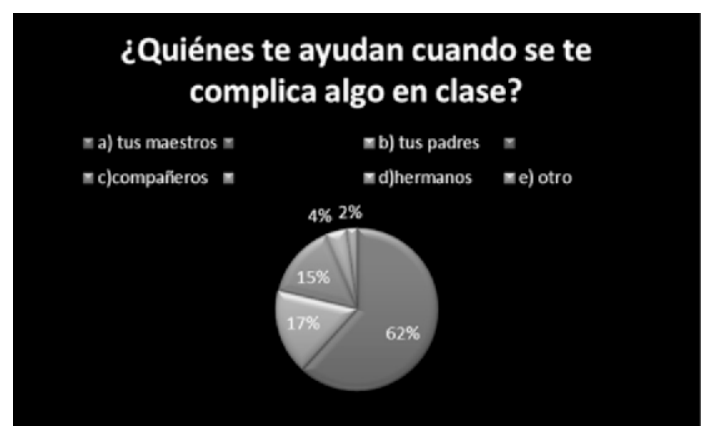

Gráfica 7
Quisiéramos hacer mención que ninguno de los encuestados, habló mal del profesor, lo cual nos indica que es de gran ayuda en el aspecto académico para los alumnos.

\section{Conclusiones}

\section{Respuesta al planteamiento del problema}

El equipo de investigación pudo comprobar que el nivel de atraso en los estudiantes de las comunidades es bajo, puesto que aproximadamente un $10 \%$ de ellos es quien presenta atraso. Se investigaron cuatro comunidades mediante muestreo no probabilístico. Se conocieron las causas del porqué los alumnos han tenido que recusar algún grado y se pudo observar que tres de cuatro niños irregulares académicamente, son conscientes de que su retraso fue por responsabilidad propia. Afirmaron que "no le echaban ganas" y son responsables de ello. Otra causa importante fue la influencia negativa por parte de sus amistades, siendo, dos casos específicos los que argumentaron que sus amistades les influenciaban para beber, a pesar de la corta edad que tenían cuando se presentó el suceso.

\section{Respuesta al objetivo general}

Se cumplió el objetivo general, aunque las expectativas del equipo eran totalmente lo contrario a lo que se descubrió. Se pudieron conocer las causas que detonan el atraso escolar. Algunas de ellas fueron falta de interés en la escuela, falta de motivación por parte del maestro e influencia negativa por parte de amistades. Se observó que las mamás de los alumnos almuerzan con ellos a la hora del receso, creando así un lazo de unión familiar, casi irrompible. Se puede inferir que existe un canal comunicativo mucho más abierto, pues las familias apoyan a los alumnos en cuanto a dificultades académicas, junto con los responsables de grupo.

También existe solidaridad entre los mismos alumnos, dando como resultado trabajos en 


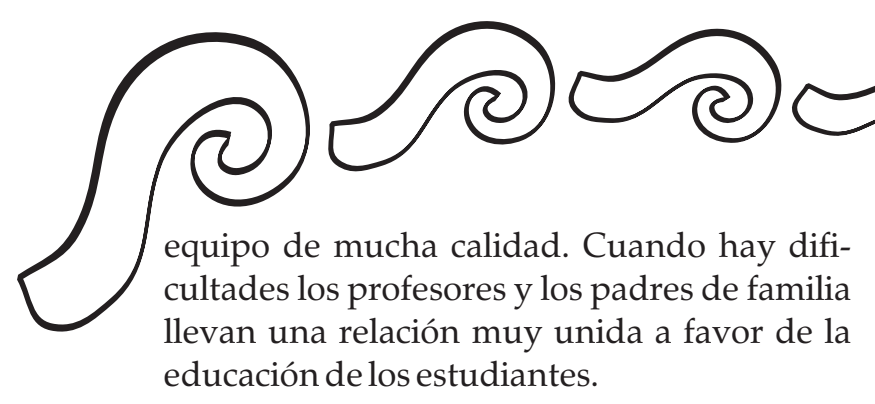

Con base en los problemas que detectamos en la ciudad, se formularon los objetivos específicos, pensando en una hipótesis de existencia de atraso escolar en las escuelas rurales multigrado. Sin embargo, podemos afirmar que hay mayor grado de atraso en las escuelas urbanas que en las rurales, al menos en las de Molango, ya que cuentan con el apoyo del Programa Federal de Oportunidades en sus familias, lo cual se percibe como la fuente principal de apoyo para ellas y los alumnos. En los pocos casos que ha habido de atraso, los maestros dan asesorías extraescolares para regularizar a dichos alumnos, o a los que necesiten apoyo extra. Las sugerencias que se pudieran aportar ya se están llevado a cabo y de forma correcta, gracias a programas de capacitación y actualización docente. Las escuelas están inscritas al programa Escuelas de Calidad, lo cual impulsa a que los directivos y maestros estén en constante mejora para dar cuentas de los resultados de sus alumnos. Al darse cuenta los alumnos que presentan atraso que ellos tienen responsabilidad en cuanto a su reprobación, es más factible que los docentes puedan ayudarles.

\section{Respuestas a las preguntas de investigación}

Falta de atención e interés por la escuela, así como mala influencia de amigos. A grandes rasgos, estas son las dos principales causas por las cuales los alumnos reprueban algún grado. Las estrategias que utilizan los maestros es dar asesorías extras para que los alumnos puedan regularizarse. Las sugerencias es que los docentes tomen talleres de actualización y sigan interesándose por sus alumnos.

\section{Limitaciones}

Las limitaciones fueron las condiciones por las que no nos podíamos trasladar a los lugares, como falta de pavimentación y carreteras.
Otra limitación fue el tiempo, puesto que la investigación se hizo como un primer acercamiento hacia las comunidades. Nos involucramos muy poco en el contexto, por la falta de tiempo y la demanda de más actividades.

La actitud introvertida de algunos niños fue limitante también porque no se pudo entrar en confianza con ellos.

\section{Aportaciones}

Este trabajo aporta al campo de la educación puesto que el atraso escolar es un problema que se viene dando desde hace tiempo y es importante trabajar para disminuirlo. Es fundamental tener en cuenta que es trabajo de todos, tanto de docentes como de padres de familia y alumnos. Fue muy enriquecedor trabajar con la gente de la comunidad de Molango y es gratificante saber que los alumnos no tienen un alto grado de atraso escolar, más bien, es esencial notar las potencialidades de los alumnos son muy elevadas y es preciso para trabajar en su desarrollo.

Este documento aporta elementos que nos sugieren analizar con mayor profundidad los prejuicios que se tienen contra la educación rural y multigrado, pues este es un ejemplo de que las condiciones académicas del campo superan aspectos existentes en las escuelas urbanas.

\section{Sugerencias}

Las sugerencias que se pueden aportar a las comunidades visitadas son las siguientes:

- Elegir a un alumno de cada grado para que sea un "alumno tutor" y que les ayude a sus compañeros a entender o explicarles la forma correcta de cómo resolver algún problema que no les sea claro si el profesor tuvo que salir o simplemente por la confianza que exista entre ellos.

- Se propone hacer talleres en las tardes para que los alumnos aprendan algu- 

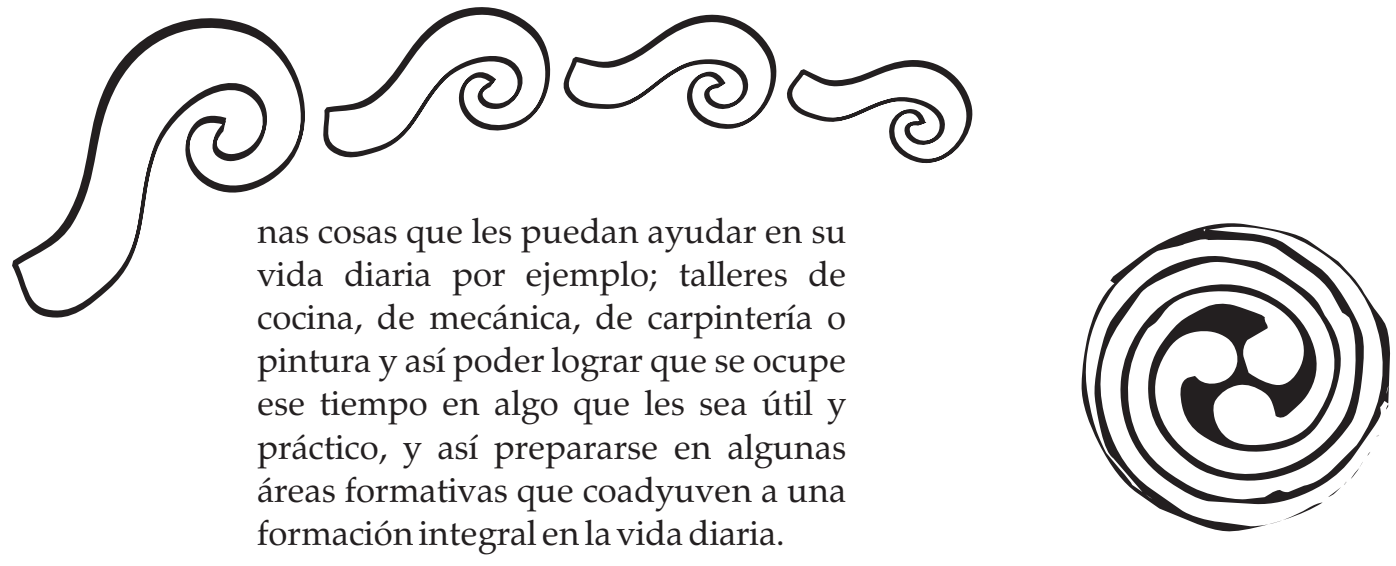

- La utilización de las TIC's en el aula, a pesar de que tienen pizarrones electrónicos y también cañones no se utilizan mucho las TIC's, pero a partir del taller que se les impartió a los profesores sobre la utilización y el buen manejo de videos, revistas e información para los alumnos podrán tener clases más innovadoras y con mayor atracción para los alumnos.
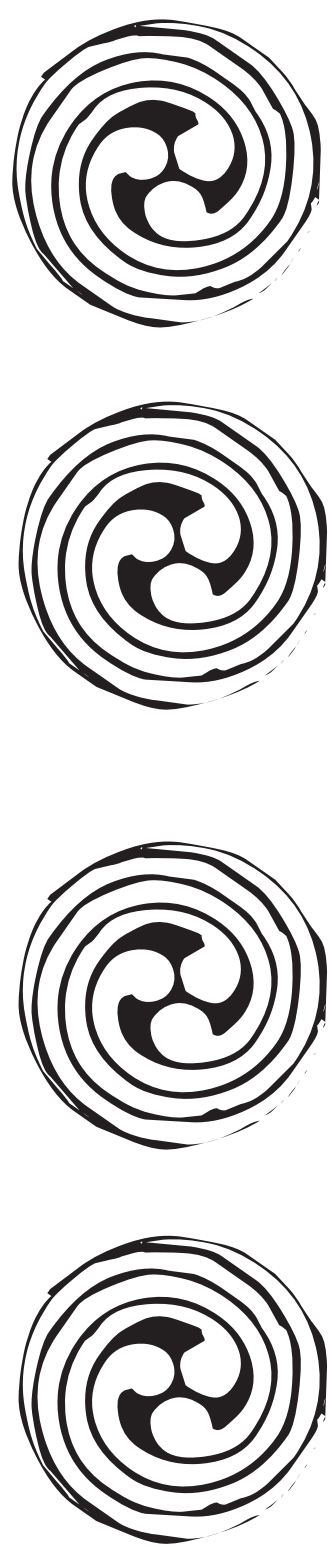


\section{Fuentes de consulta:}

Diccionario electrónico definición de (s.f.) Definición de. En línea: http://definicion.de/rural/ Consultado el 11 de octubre de 2011.

Equipo Regional de Misión Educativa (2007) Proyecto Educativo Regional Lasallista Latinoamericano. León, Guanajuato, México.

Farlex, (s.f.) The Free Dictionary. Diccionario electrónico. En línea: http://es.thefreedictionary.com Consultado el 11 de septiembre de 2011

Gallardo Gutiérrez, Ana Laura (s.f.) Las escuelas multigrado frente a la diversidad cultural, étnica y lingüística. Horizontes de posibilidad desde la educación intercultural. Artículo publicado en internet. En línea: www.amdh.com.mx/ocpi/documentos/ docs/6/20.docConsultado el 11 de octubre de 2011.

Granja, Castro, Josefina (2009) Las categorías de la escolarización: el concepto de retraso escolar en los inicios del siglo XX. Artículo publicado en internet. En línea: http://www.comie.org.mx/congreso/memoria/v10/pdf/area_tematica_09/ponencias/1199F.pdf. Consultado el 10 de octubre de 2011

Lavín de Arrivé, Sonia (2005) Centro de investigación básica intensiva: una alternativa al rezago escolar Artículo publicado en internet de la revista Redalyc. En línea: http://redalyc.uaemex.mx/pdf/270/27035403.pdfConsultado el 10 de septiembre de 2011.

Nuestro México (s.f.) Pemuxtitla. Artículo publicado en internet. En línea: http://www.nuestro-mexico.com/Hidalgo/Molango-de-Escamilla/Areas-de-menos-de-500habitantes/Pemuxtitla/Consultado el 16 de noviembre de 2011

Psicopedagogia.com (s.f.) Retraso escolar. Artículo publicado en internet. En línea: http://www.psicopedagogia.com/retraso-escolar Consultado el 11 de septiembre de 2011.

Pueblos américa, (s.f.)Tenango. Artículo publicado en internet. En línea http://mexico.pueblosamerica.com/i/tenango-4/Consultado el 16 de noviembre de 2011

Sacristán, J. Gimeno (1988) El currículum: una reflexión sobre la práctica. Ed. Morata, S.L., Madrid, España

Secretaría de Educación Pública (2011) La creación de la SEP. Portal oficial. En línea: http://www.sep.gob.mx/es/sep1/sep1_Historia_de_la_SEP Consultado el 10 de septiembre de 2011

Vázquez, Josefina Zoraida (2005) La educación en la historia de México. Artículo recuperado de la compilación del Colegio de México. Ed. El Colegio de México. México, D.F., México. http://www.red-ler.org/cambiar-escuela-rural.pdf el11 de septiembre de 2011.
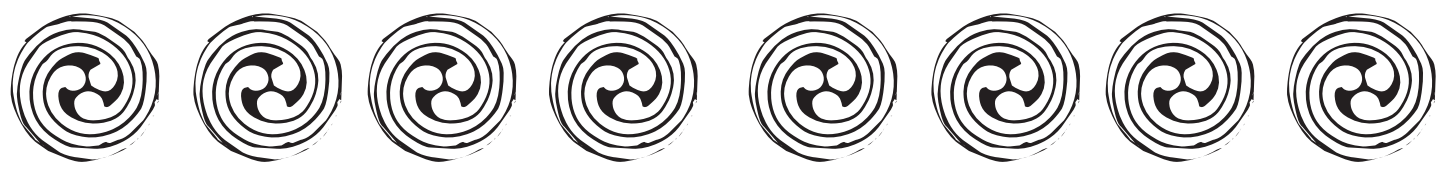

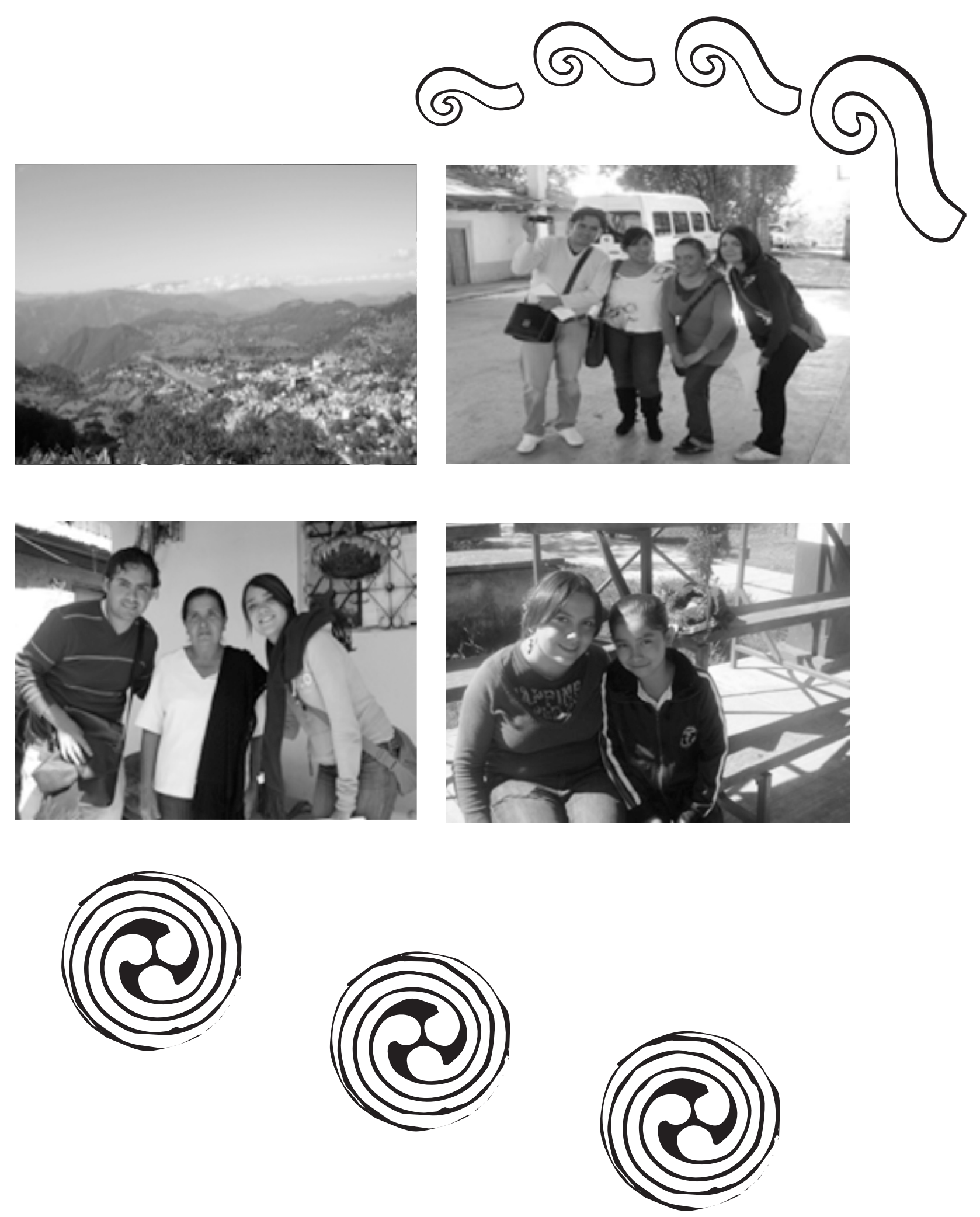